\title{
Moving Target Detection and Doppler Extraction Using Digital Spread Spectrum Radar
}

\author{
Mr. Subhankar Shome \\ Sikkim Manipal Institute of Technology, Sikkim Manipal University, East Sikkim, India \\ subho.ddj@gmail.com \\ Dr. Rabindra Nath Bera \\ Sikkim Manipal Institute of Technology, Sikkim Manipal University, East Sikkim, India \\ rbera50@gmail.com \\ Mr. Samare ndra Nath Sur \\ Sikkim Manipal Institute of Technology, Sikkim Manipal University, East Sikkim, India \\ samar.sur@gmail.com \\ Mr. Rabi Adhikary \\ Surendra Institute of Engineering \& Management, WBUT, West Bengal, India
}

\begin{abstract}
In this paper, we discuss a Spread Spectrum based radar system for car detection in the road and autonomous guidance of vehicles. An autonomous intelligent vehicle has to perform a number of functionalities. Segmentation of the road, determining the boundaries to drive in and recognizing the vehicles and obstacles around are the main tasks for vision guided vehicle navigation. In this article we propose a set of algorithms which lead to the solution of road and vehicle collision using carrier recovery method from car velocity using DSSS RADAR. In such a spread spectrum system, the transmitted signal is spread over a larger bandwidth, which is much wider than the minimum bandwidth required to trans mit the information. Automotive radar systems can take advantage of spread spectrum techniques because of their interference rejection, immunity from noise and multipath distortion, and high resolution ran ging properties. In addition, there is no need for high-speed, fast-settling frequency synthesizers. Moreover, spread spectrum techniques can improve the reliability of automotive radars. The data from different sensors on different cars can be combined in order to observe the complete car environment. Thus a spread spectrum radar system will allow sharing the same bandwidth also for data link needed by car-tocar communication systems. The algorithm described here is to recover Doppler frequency using $2 \mathrm{P}$ power method from which we are able to detect the vehicle condition in road.
\end{abstract}

Index Terms - DSSS, Doppler, 2P Power method, P4

\section{INTRODUCTION}

Doppler radar is specialized radar that makes use of the Doppler effect [1] to produce velocity data about moving objects at a distance. Doppler radar [2] does this by beaming a microwave signal towards a desired target and listening for its reflection, then analyzing how the frequency of the returned signal has been altered by the object's motion. This variation gives direct and highly accurate measurements of the radial component of a target's velocity relative to the radar. Doppler radars are used in aviation, sounding satellites, meteorology, police speed guns, and radiology.

Doppler radars [3] use Doppler effect, which is a frequency variation due to distance change of moving objects. There are three ways of producing the Doppler Effect. Radars may be Coherent pulsed (CP), Continuous wave (CW), or Frequency modulated (FM)- CW [4] Doppler radar only measures a velocity output while frequency modulated continuous wave(FM-CW) radar measures speed (velocity) and distance (range). In a spread spectrum system the transmitted signal is spread over a frequency band, which is much wider than the minimum bandwidth required transmitting the information.

Automotive radar systems can take advantage of spread spectrum techniques because of their interference rejection, immunity from noise and multipath distortion, and high resolution ranging properties. In addition, there is no need for high-speed, fast-settling frequency synthesizers. Moreover, spread spectrum techniques can improve the reliability of automotive radars. The data from different sensors on different cars can be combined in order to observe the complete car environment. Thus a spread spectrum radar system will allow to share the same bandwidth also for data-link needed by car-to-car communication systems.

To simu late this type of Spread Spectrum RADAR for Intelligent Transport System the authors have introduced the DSSS Technology [5]. The implementation of the DSSS Radar system involved the analysis and design of unique synchronization loops goal is to simu late the usual appearance of papers in a Journal of the Academy Publisher. We are requesting that you follow these guidelines as closely as possible. for code tracking and carrier estimation, as a result of complex spreading sequences. 
The SS Communications are widely used today for Military, Industrial, Avionics, Scientific, and Civil uses. The advantages of using SS include the following.

- Low power spectral density.

As the signal is spread over a large frequency-band, the Power Spectral Density is getting very small, so other communication systems do not suffer from this kind of communication.

The ability to utilize the Satellite payload channels, which is achievable as the transmitted signal is spread in such a way that it becomes noise-like and thus would not interfere with the payload traffic.

- Interference limited operation.

- Privacy due to unknown random codes.

- Applying spread spectrum implies the reduction of multi-path effects.

- Random access possibilities. As users can start their transmission at any arbitrary time.

- Good anti-jamming performance.

Those unique features of spread spectrum help authors to build an advanced radar receiver that is capable to detect Doppler shift due to the velocity of car. The developed systems pecification is as follows:

$\begin{array}{lll}\text { i. } & \text { Radar Signal } & \text { : Spread Spectrum } \\ \text { ii. } & \text { Code } & \text { : Polyphase } \\ \text { iii. } & \text { Bandwidth } & : 100 \mathrm{MHz} \\ \text { iv. } & \text { IF } & : 70 \mathrm{MHz} \\ \text { v. } & \text { RF } & : 3 \mathrm{GHz}\end{array}$

The rest of the paper is organized as follows. The mathematical model of the system is described in section II. In section III, MATLAB platform based Radar simulation is described. The Hardware description of the system is presented in section IV. Section V contains the experimental result and finally section VI concludes the paper.

\section{MATHEMATiCAL MOdEL OF ThE System}

\section{A. Doppler Spread due to car velocity.}

To have a theoretical model [6] of Doppler spread we assume that transmitted signal ST(t) is a constant envelop phase modulated signal given by

$$
\mathrm{ST}(\mathrm{t})=\mathrm{A} \cdot \exp [\mathrm{j}(\omega \mathrm{t}+\psi \mathrm{s}(\mathrm{t}))]
$$

Where $\mathrm{A}$ is a constant, $\omega$ is the angular radio frequency, $\psi s(t)$ is the information-bearing baseband signal. We can also model the time varying random propagation medium having the transfer function $\mathrm{p}(\mathrm{t})$ given by

$$
\mathrm{p}(\mathrm{t})=\mathrm{r}(\mathrm{t}) \exp [\mathrm{j} \psi \mathrm{r}(\mathrm{t})]
$$

Where $r(t)$ is the time-variable envelope of $p(t)$ and $\psi s(t)$ the time-variable random phase of the propagation mediu $\mathrm{m}$. The envelop of the random propagation mediu $\mathrm{m}$ $\mathrm{r}(\mathrm{t})$ can be separated into long-term or average fad ing $\mathrm{m}(\mathrm{t})$ and short term or fast multipath fading ro(t) parts defined by

$$
r(t)=m(t) \cdot r o(t)
$$

Considering the model of propagation medium to be mu ltip licative fading channel, the received signal SR(t) at the receiving end is the product of $\mathrm{ST}(\mathrm{t})$ and $\mathrm{p}(\mathrm{t})$ and can be expressed as

SR (t) $=\mathrm{ST}(\mathrm{t}) \cdot \mathrm{p}(\mathrm{t})=\mathrm{A} \cdot \exp [\mathrm{j}(\omega \mathrm{t}+\psi \mathrm{s}(\mathrm{t}))]$. $r(t) \exp [j \psi s(t)]=A \cdot \exp [j(\omega t+\psi s(t))] \cdot m(t) \cdot r o(t) \cdot \exp [j \psi r(t)]$

$$
\mathrm{SR}(\mathrm{t})=\mathrm{A} \cdot \mathrm{m}(\mathrm{t}) \cdot \operatorname{ro}(\mathrm{t}) \cdot \exp [\mathrm{j}(\omega \mathrm{t}+\psi \mathrm{s}(\mathrm{t})+\psi r(\mathrm{t}))]
$$

Here random-phase varying term $\psi \mathrm{r}(\mathrm{t})$ in the expression of SR(t) given by (5) is the cause of Doppler spread. This random phase variation induces random frequency modulated (FM) noise at the receiver.

\section{B. Spectral Matched Filter for Correlation}

The algorithm can be enhanced to provide better estimation accuracies at higher chip rates. The enhancement is to spectrally match filter the averaged periodogram prior to the peak search.

The matched filter coefficients are based on the power spectral density (PSD) of the spread-spectrum, binary phase shift keying (BPSK) signal used in DAMA. With this filtering, which serves to enhance the averaged periodogram, we are able to accurately estimate carriers which have higher PGs (which is desirable). The timedomain matched filter (MF) is a linear filter designed to provide the maximum signal-to-noise power ratio at its output for a given transmitted symbol waveform. It can be shown that the time domain MF is given by

$$
h(t)= \begin{cases}s(T-t), & 0 \leq t \leq T \\ 0 & \text { elsewhere }\end{cases}
$$

where $s(t)$ is the known signal and $T$ is the symbol time duration. Here the time-domain MF impulse response is the time-reversed (and shifted for causality) signal. Threshold comparison is then made on $\begin{array}{llllll}r & t & h & t & \text { where } & r(t)\end{array}$ and $*$ indicates the convolution operation.

The MF idea may also be used to provide a maximum spectrum-to-noise power ratio. In this case we take the frequency-domain analog to (4) by first computing the power spectraldensity (PSD),

$$
\begin{gathered}
P_{x}\left(e^{j \omega}\right) \text { of our known process } x(n) \\
p_{x}\left(e^{j \omega}\right)=\sum_{n=-\infty}^{\infty} r_{x}(n) e^{-j \omega n}
\end{gathered}
$$

Where the correlation sequence is given by

$$
r_{x}(n)=E[x(k) x(k-n)]
$$

and $E[]$ indicates the expectation operator.

\section{2P-Power Methodfor Doppler Recovery:}

We return to AWGN channel and concentrate on nondata aided phase estimators. Under the assumption of ideal timing and carrier frequency recovery, we investigate two methods. The first is suitable for full response formats while the other applies to MSK type signaling. 
We begin with the signal model

$$
s(t)=e^{J \theta} \sqrt{\frac{2 E_{s}}{T}} e^{j \psi(t-\tau . \alpha)}
$$

Where

$$
\psi(t, \alpha)=2 \pi h \sum_{i} \alpha_{i} q(t-i T)
$$

With full response modulation the phase response satisfies relations

$$
q(t)= \begin{cases}0, & t \leq 0 \\ 1 / 2, & t \geq T\end{cases}
$$

Thus, taking $t=-i_{0} T$ as the starting time and letting $\mathrm{h}=\mathrm{K} / \mathrm{P}$, from (1.2) we get

$$
2 P \psi(k T)=2 \pi K \sum_{i=-i_{0}}^{k-1} \alpha_{i}
$$

Which indicates that $2 P \psi(k T)$ is a multiple of $2 \pi$.

$$
S^{2 P}(k T+\tau)=\left(\frac{2 E_{s}}{T}\right)^{P} e^{j 2 P \theta}
$$

Clearly, the sequence $\left\{s^{2 P}(k T+\tau)\right\}$ contains information on the carrier phase. Unfortunately we have no direct access to $\mathrm{s}(\mathrm{t})$ but only to the received waveform $\mathrm{r}(\mathrm{t})$. Nevertheless, the latter can be filtered (to limit excess noise) and used in place of $s(t)$. Denoting $\mathrm{x}(\mathrm{t})=\mathrm{s}(\mathrm{t})+\mathrm{n}(\mathrm{t})$ the filtered version of $\mathrm{r}(\mathrm{t})$ and letting $x(k) \underline{\underline{\Delta}} x(k T+\tau)$ produces

$$
x^{2 P}(k)=\left(\frac{2 E_{s}}{T}\right)^{P} e^{j 2 P \theta}+N(k)
$$

Where $\mathrm{N}(\mathrm{k})$ is a zero-mean random term resulting from the products SignalXNoise and NoiseXNoise in the binomial expansion of $(s+n)^{2 P}$.

At this point a phase estimation algorithm is easily conceived.

Hence, sampling s (t) at $t=k T+\tau$ and rising to the $2 \mathrm{P}$ th Power yields.

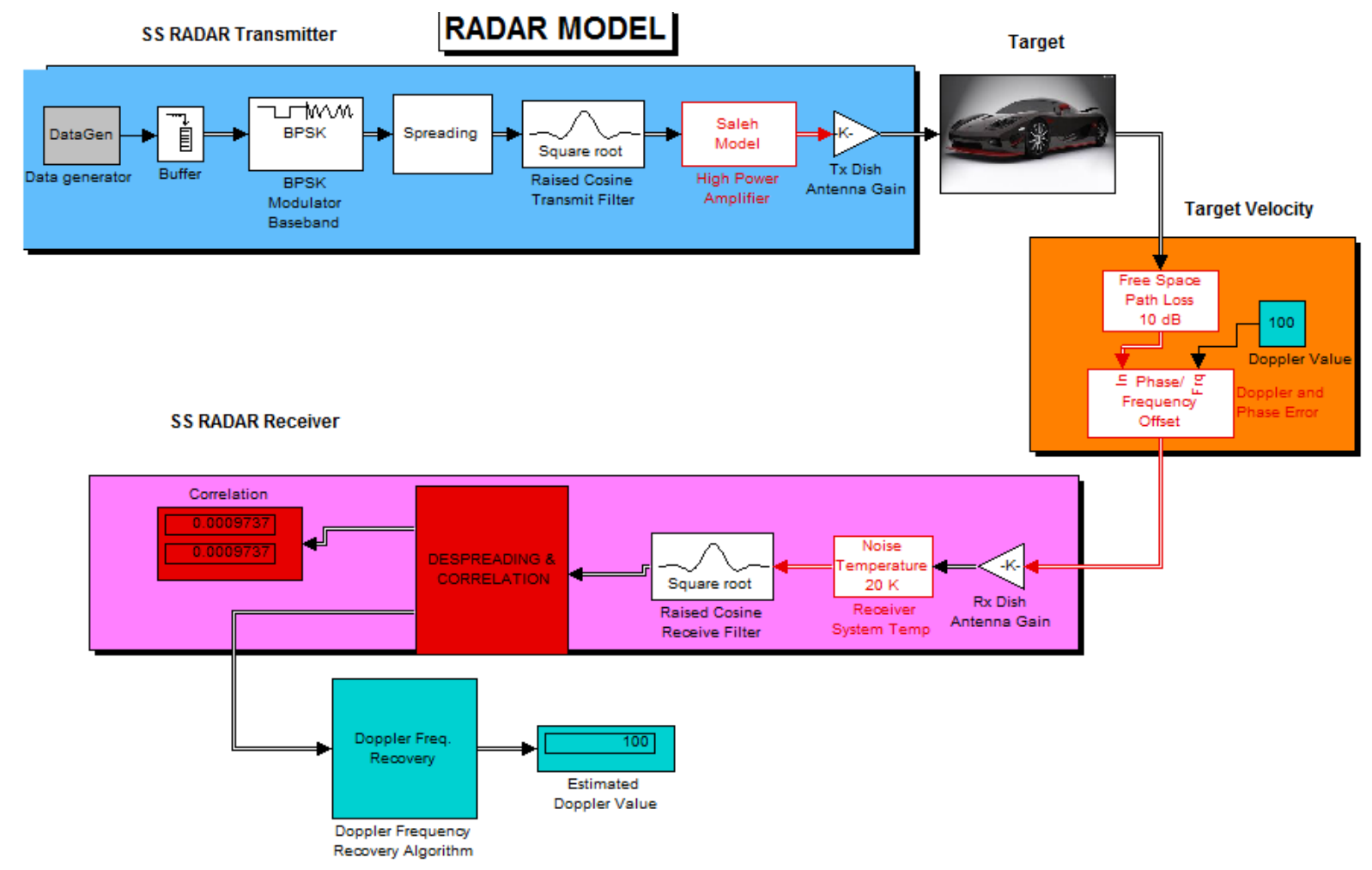

Fig. 1. Matlab simulation of SS Radar for Target detection and Doppler recovery

\section{MATLAB SIMULATION}

Fig. 1 shows the simulated block diagram [7] of the SS Radar for Target detection and Doppler recovery [8]. In transmitter side a data generator is used as input data source. BPSK modulator maps the data stream into two constellations. Here spreading is introduced as second level modulation scheme, in order to achieve high security and anti-jamming capability in Radar system. Polyphase Code [9] is used for Spreading operation. Raised Cosine Transmit Filter is used to up-samples and shapes the modulated signal using the square root raised cosine pulse shape. After pulse shaping authors are used Saleh Model as travelling wave tube amplifier (High Power Amplifier). Gain block to introduce the Tx. 
Antenna Gain., for RADAR operation. , the TARGET block has been modeled based on the RADAR Range equation. This Spread Spectrum based transmitted signal is reflected back from the target body, as here it is considered as a Car on the road. Phase and Frequency offset block will introduce the Doppler effect due to the car velocity. TARGET and target velocity block together will create a situation of a moving object on the road. The signal reflecting back from a moving object will have some Doppler information in it, which is shown as 100 $\mathrm{Hz}$ in our simulation and this value will be extracted in the receiver side. Simulated block diagram of the designed radar receiver, receives the signal using $\mathrm{Rx}$. Antenna Gain of the receiver parabolic dish antenna at the ground station. Raised Cosine Receive Filter applies a matched filter to the modulated signal using the square root raised cosine pulse shaping.
A spread spectrum receiver [10] uses a locally generated replica of the transmitter code in receiver correlator to separate out the desired coded information from all possible signals with proper time synchronization. A spread spectrum correlator can be thought of as a very special matched filter -- it responds only to signals that are encoded with a code (here polyphase code) that matches its own code. Thus, an SS correlator can be "tuned" to different codes simply by changing its local code. This correlator does not respond to manmade, natural or artificial noise or interference [11].

The proper correlation of the transmitted and received signal results in maximum correlation peak, which will provide us the information about target detection by Radar receiver [12].

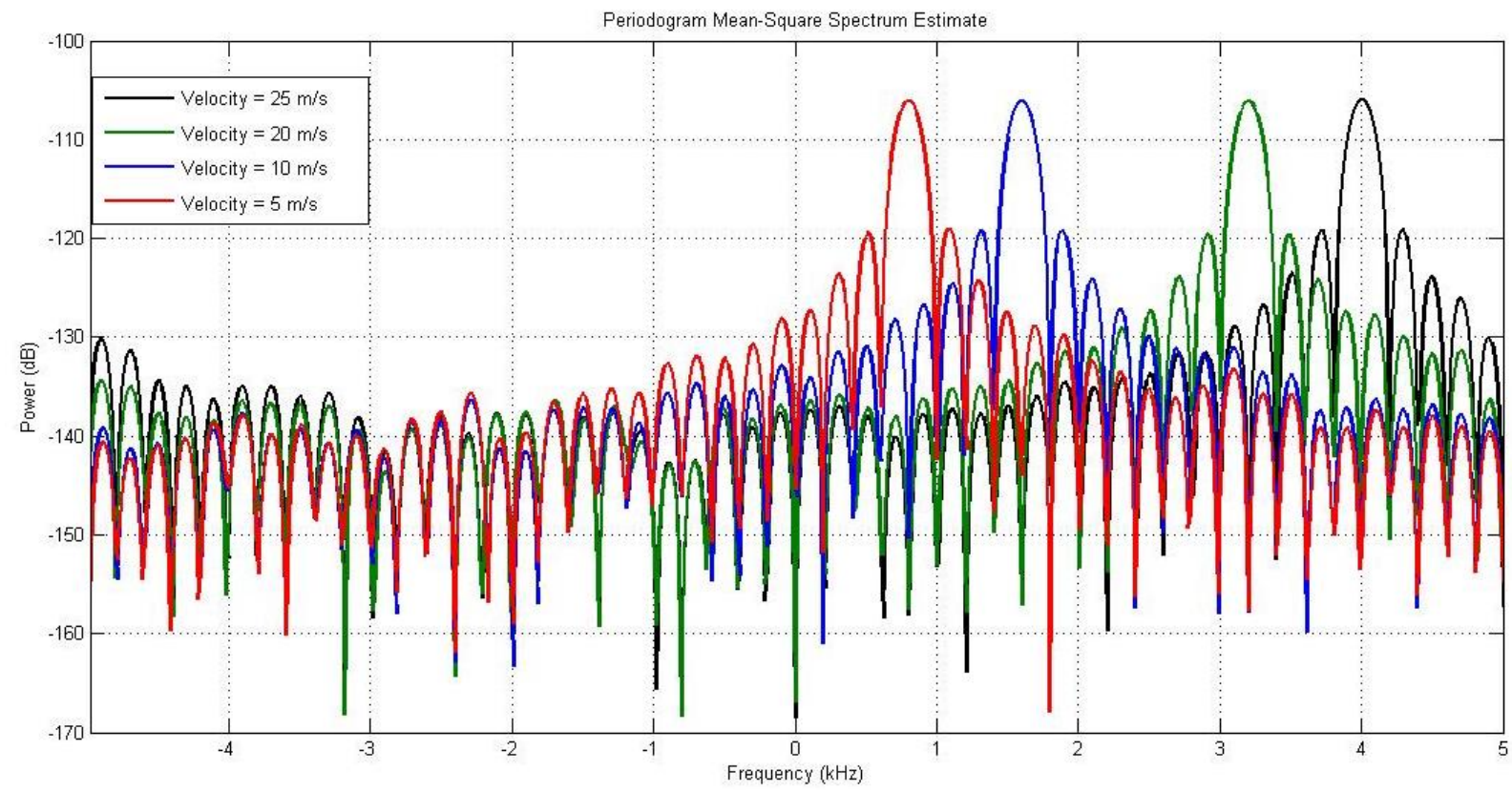

Fig. 2. Doppler shift due to target velocity.

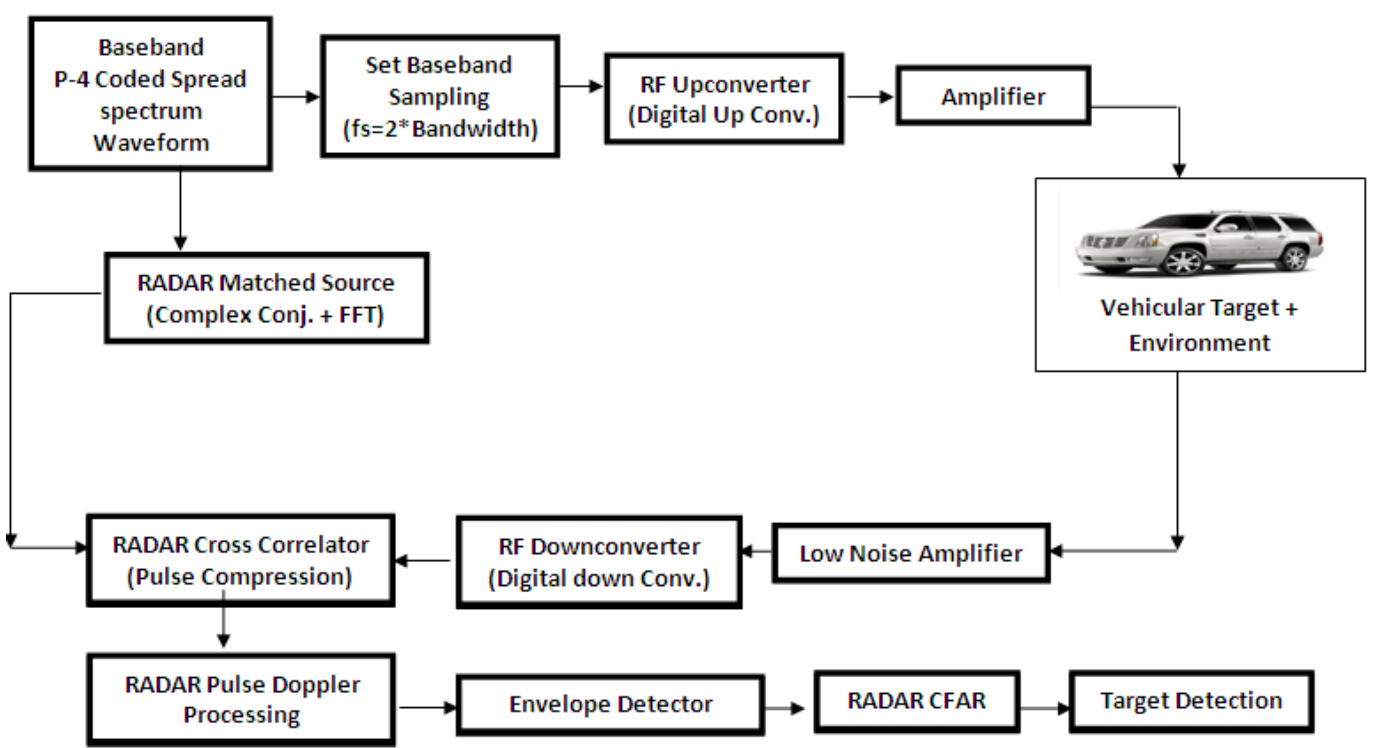

Fig. 3. Developed system schematic 
The mathematical part of the correlator based on special Matched filtering is discussed in mathematical modeling part in details. After this block we have used the Doppler recovery block based on $2 \mathrm{P}$ power method, which is basically giving us the information about Target velocity. Here also we have discussed the detail algorith $m$ in mathematical modeling part.

Figure 2, represent Doppler due to different target velocity like: $5 \mathrm{~m} / \mathrm{s}, 10 \mathrm{~m} / \mathrm{s}, 20 \mathrm{~m} / \mathrm{s}$ and $25 \mathrm{~m} / \mathrm{s}$ which are estimated at the receiver and based on the correlator receiver with $2 \mathrm{P}$ method.

\section{DESCRIPTION OF HARDWARE SYSTEM}

Figure 3 shows the block diagram of the designed radar hardware system. Baseband Signal generation is based on AWG (Arbitrary Waveform generator). P4 code is used for spreading operation.

$\mathrm{P} 4$ codes are beneficial in modern radar, having MPSK (M-ary Phase Shift Keying) property, and are normally derived from the phase history of Frequency-modulated pulse.

P4 codes can be expressed as:

$\varphi_{\mathrm{i}}=\pi(\mathrm{i}-1)(\mathrm{i}-1-\mathrm{M}) / \mathrm{M}$,

where

$\varphi_{\mathrm{i}}=\mathrm{i}$ th order phase.

$\mathrm{M}=$ no of phase states

The values of $\varphi_{i}$ are computed following equation 1 and saved in one file which is subsequently downloaded tom AW G (Arbitrary Waveform generator) for hardware realization of the $\mathrm{P} 4$ code.
This waveform will be modulated over IF carrier of $70 \mathrm{MHz}$ and subsequently up-converted to RF carrier between 0.3 to $3.0 \mathrm{GHz}$. Here we have used 25 bits $\mathrm{P} 4$ code

\section{A. Transmitter}

\section{- Baseband Code Generation:-}

Baseband Transmitted Signal of the Radar System mainly consists of pulse generation and $\mathrm{PN}$ sequence generation. Polyphase code is used in SS Radar operation. The pulse with required width is multiplied by the Polyphase code for having desired duty cycle.

\section{- IF Section:-}

In this subsystem, the baseband signal is modulated with the intermediate frequency (IF) of $70 \mathrm{MHz}$ with the help of a mixer. Both the baseband and IF sections will be realized using an Arbitrary Waveform Generator (AWG) which utilize the Digital generation scheme. Thus at the output of the AWG we have $70 \mathrm{MHz}$ dig ital modulated pulsed Polyphase code. The baseband and IF sections are realized in AWG.[13]

\section{- RF Section:-}

The output of the AWG is being fed to the RF subsystem using a $15 \mathrm{~m}$ length Coaxial cable. In RF Subsystem, the signal is up-converted to RF level using RF mixer with the local oscillator frequency stepped from 0.3 to $3 \mathrm{GHz}$ with $5 \mathrm{MHz}$ frequency step (using Vector Signal Generator (VSG)). The RF signal is then amplified using power amplifier and transmitted using Antenna.

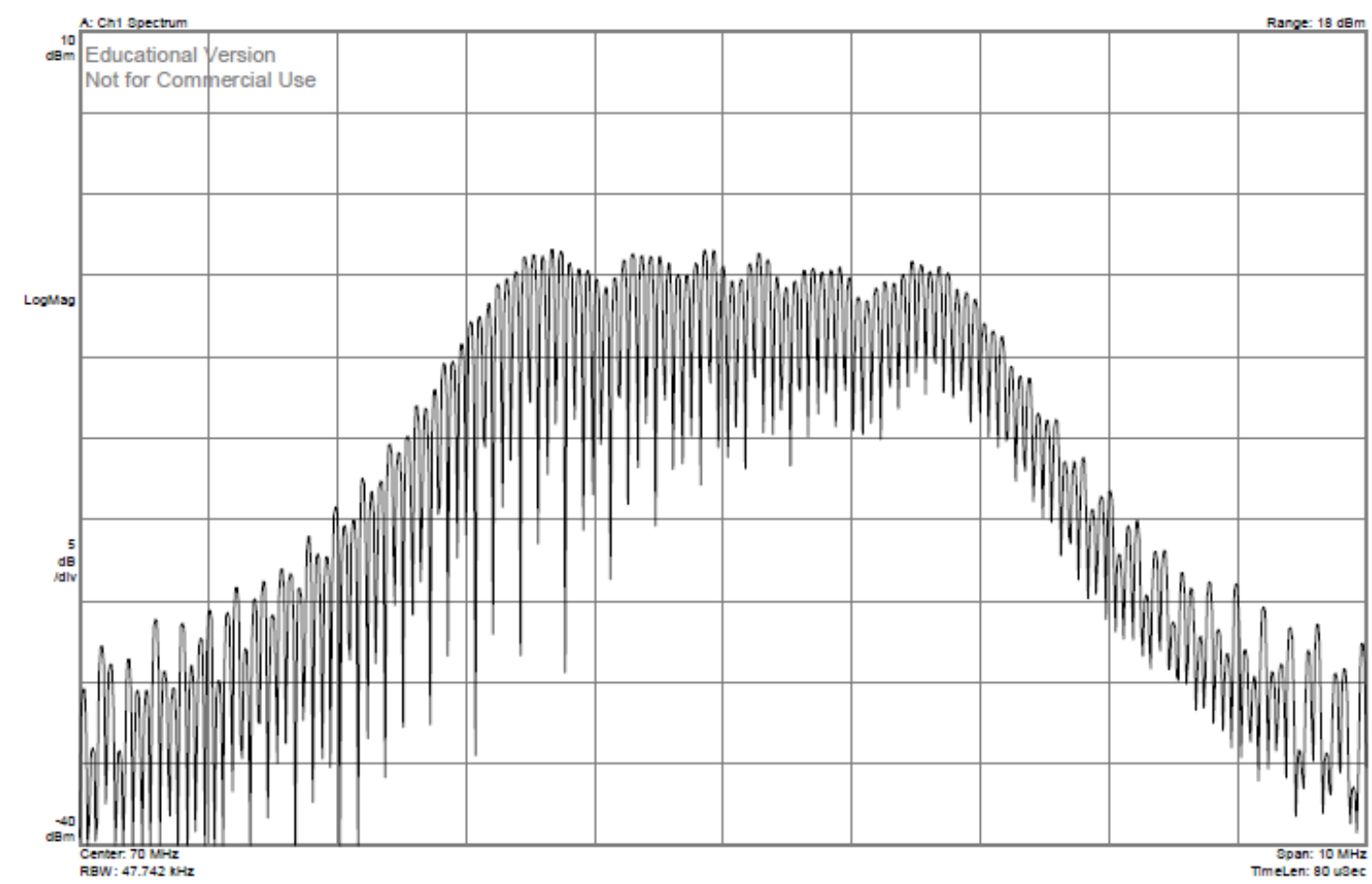

Fig. 4. Typical P4 code modulated IF spectrum

\section{B. Receiver}

- Receiver front end:- 
At the receiver end, the received signal from the target will be received using a separate receive antenna and then fed to a Low Noise Amplifier. The amplified signal is down converted, using the local oscillator frequency generated by the same Vector Signal Generator (VSG), to the IF level (i.e. $70 \mathrm{MHz}$ ). A single VSG is thus used in this way to synchronize both TX and RX systems.

\section{- Agilent VSA U1065A as Receiver}

Agilent VSA [14] is a versatile radar signal analy zer of a new kind of Instruments fully programmable via its own embedded Agilent 89600 VSA software or user programmable from PC through SYSTEMVUE or MATLAB.

This is used as programmable receiver providing a maximum sampling rate of $8 \mathrm{GS} / \mathrm{s}$.. The VSA has additional in-built function of Correlation filter which is best utilized for noise and clutter reduction in radar operation.

Correlation processing of target identification and delay calculation will be done in VSA. Thus VSA will be one of the critical equipment for the said SS RADAR design.

\section{Frequency stepping and Receiver Gating}

The VSG will generate RF signal in frequency range of 0.3-3 GHz and this signal will be used to up convert the IF level signal and down convert to IF level in transmitting and the receiving section respectively. The instantaneous bandwidth of the radar will be $5 \mathrm{MHz}$ and to cover a total bandwidth from $0.3 \mathrm{GHz}$ to $3 \mathrm{GHz}$, the RF frequency will be stepped with a step size of $5 \mathrm{MHz}$. To operate the Radar in bi-static mode the RF over Optical transmitter and receiver (TOR/ROT) will be used to transmit the frequency stepped signal [15]. The receiver gating will be controlled by CPG2. The Delay in receiver gating operation will be varied manually and set to the optimum position to define only the target zone.

\section{EXPERIMENT AL RESULT}

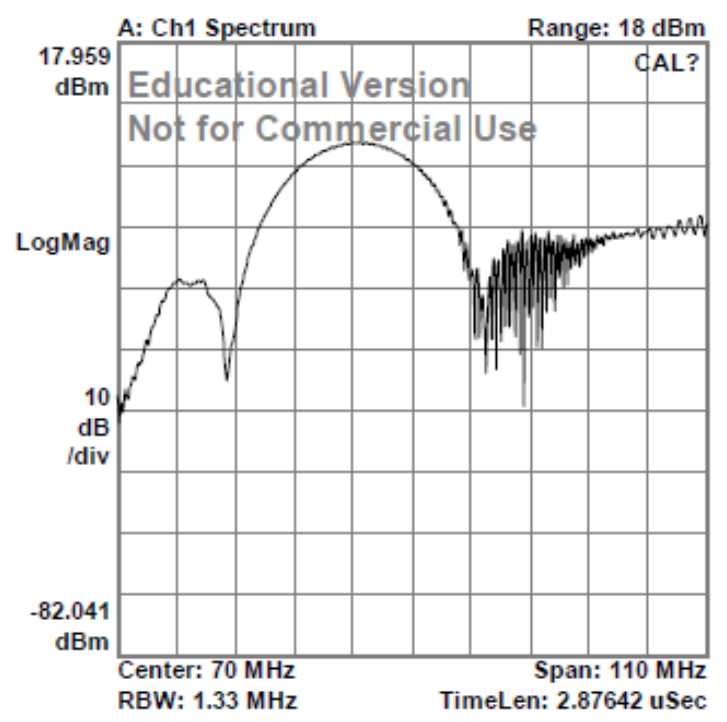

Fig. 5. Transmitted $70 \mathrm{MHz}$ IF Signal

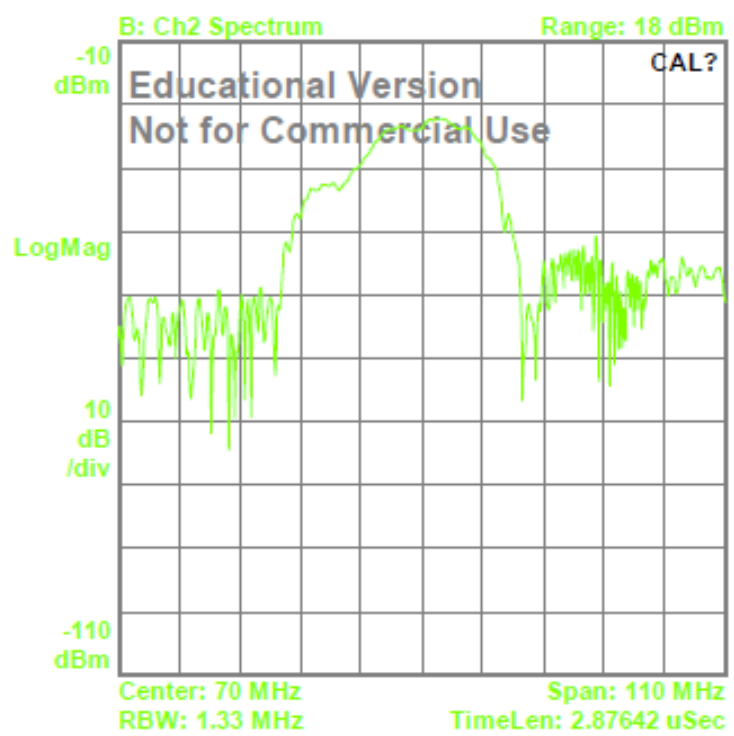

Fig. 6. Received IF signal

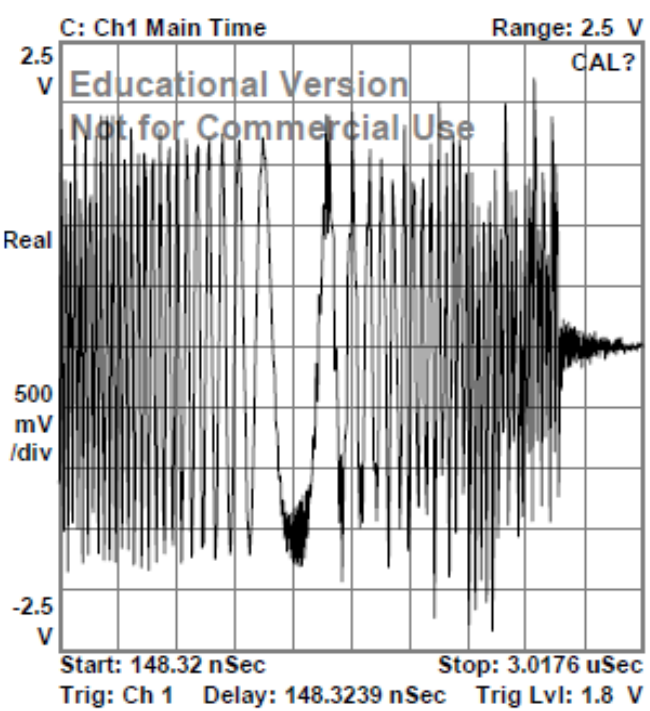

Fig. 7. Transmitted $\mathrm{P} 4$ Code

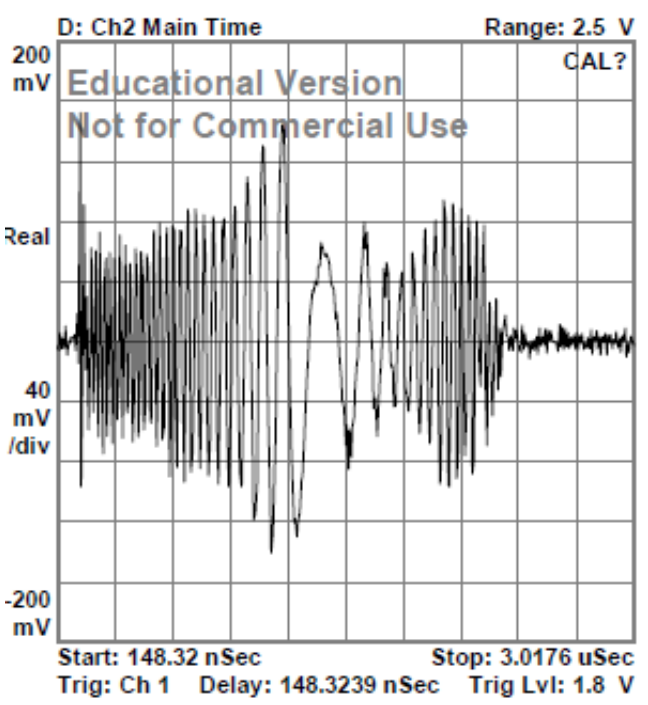

Fig. 8. Received P4 Signal 


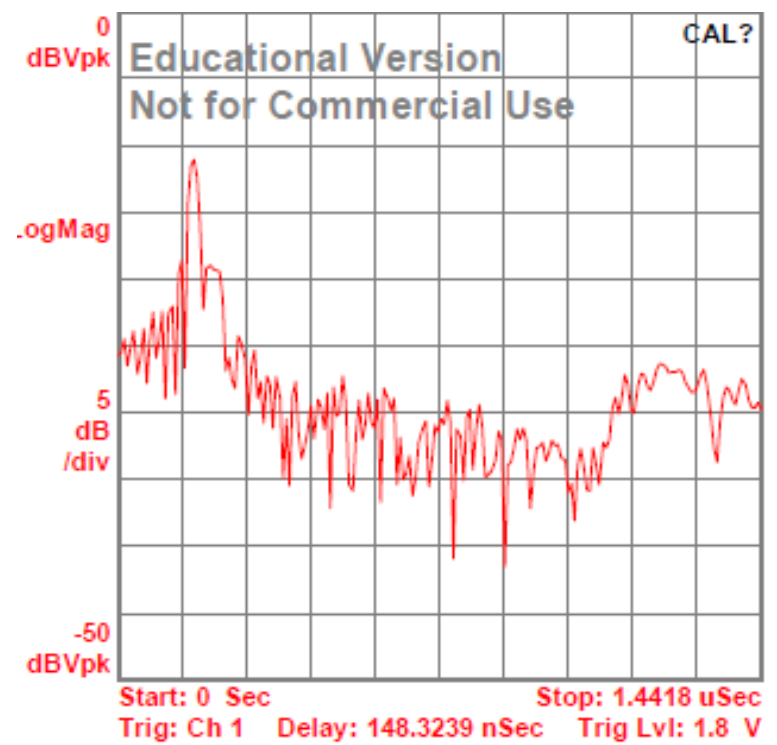

Fig. 9. Correlated output or Target detection

\section{CONCLUSION}

Recent automobile radars from both Japanese and other manufacturers are commercially available. There are a variety of modulation methods for such Millimeter wave automobile radar, such as FM-CW, 2-frequency $\mathrm{CW}$, pulsed LFM, and Spread Spectrum (SS). However, FMCW (Frequency-Modulated Continuous Waves) is utilized most often, since the high-frequency band has a comparatively simple structure, it can measure both distance and range rate simultaneously with a high degree of precision and it can also measure range rates of zero. Pulsed LFM radar is very popular for Smart Vehicle Application. Accordingly Digital Chirp radar in the form of SS technology are also evolving for ITS [Intelligent Transport System] application. But, this is relatively new technology having lots of added advantages over LFM waveforms. To Prove a SS Radar authors tried to prove the concept in simu lation first, after getting a satisfactory result in SIMULINK model, authors designed a SS Radar System in hardware based on AWG, VSA along with up conversion and down conversion circuit. At this stage authors have proven the concept of SS Radar in actual hardware and detected static as well as moving target like cars on road, which may be very much useful for collision avoidance in near future. Authors are als o trying to use the target detected information or data to use for Target imaging in next step, which will add an another dimension for SS radar and will proved a better road scenario for automated car as sistance systems.

\section{REFERENCES}

[1] Doppler Radar Speed Measurement Based On A $24 \mathrm{GHz}$ Radar Sensor, Joacim Dybedal, Norwegain University of Science and Technology

[2] http://en.wikipedia.org/wiki/Doppler_effect

[3] G. Eason, B. Noble, and I. N. Sneddon, "On certain integrals of Lipschitz-Hankel type involving products of
Bessel functions," Phil. Trans. Roy. Soc. London, vol. A247, pp. 529-551, April 1955. Doppler weather radar based nowcasting of cyclone Ogni by Soma Sen Roy 1, V Lakshmanan2, S K Roy Bhowmik1 and S B Thampi3. 1 India Meteorological Department, Lodi Road, New Delhi 110003 , India. 2 National Severe Storms Laboratory \& University of Oklahoma, Norman, Oklahoma 73069, USA 3 Doppler Weather Radar Station, India Meteorological Department, Chennai, India.

[4] Griffiths, H.D.: New ideas in FM radar, Electronics Communication Engineering Journal, vol.2, no.5, pp.185194, Oct 1990

[5] Kandar D and Bera R (2005) Development of a imaging RADAR instrumentation system using DS-UWB wireless technology. Proc. of XXVIII ${ }^{\text {th }}$ general assembly of int. union of radio science (URSI).

[6] Signal Processing Methods for Doppler Radar Heart Rate Monitoring. By Anders Høst-Madsen1,2, Nicolas Petrochilos2, Olga Boric-Lubecke1,2, Victor M. Lubecke1,2, Byung-Kwon Park2, and Qin Zhou3 Kai Sensors, Inc., HI, USA1, University of Hawaii at Manoa, HI, USA 2 and Broadcom, Inc., USA3

[7] Mathworks Simulink model of RF satellite link \& WCDMA phy sical Layer

[8] Purdy, R. J., Blankenship, P. E., Muehe, Ch. E., Rader, Ch. M., Stern, E., Williamson, R. C.: Radar Signal Processing, Lincoln Laboratory Journal, vol.12, no.2, pp.297-320, 2000

[9] SIDE LOBE SUPRESSION TECHNIQUES FOR POLYPHASE CODES IN RADAR, A THESIS SUBMITTED IN PARTIAL FULFILLMENT OF THE REQUIRMENT FOR THE DEGREE OF, Master of Technology in Telematics and Signal Processing By VIJAY RAMYA KOLLI

[10] Spread Spectrum Digital Beamforming (SSDBF) Radar, by Dr. Marcos A. Bergamo. Applied Radar Inc. 200 Wheeler Road Burlington, MA 01803, USA.

[11] S. Sayama, M. Sekine, "Amplitude statistics of ground clutter included town using a millimeter wave radar," IEICE Trans. Commun (Japanese Edition)., vol.J86-B, no.5, pp.829-836, May 2003.

[12] Isamu Matsunami, Youichiro Nakahata, Katsushi Ono, Yuusei Noguchi and Akihiro Kajiwara, " Empirical Study on Ultra-Wideband Vehicle Radar", Vehicular Technology Conference, 2008. VTC 2008.

[13] http://www.home.agilent.com/en/pc-2338313/high performance-arbitrary-waveform-generator?nid=-32928.0 \&cc= IN\&lc=eng

[14] http://www.home.agilent.com/en/pc-1905089/89600-VSA -and-WLA-Software?\&cc=IN\&lc=eng

[15] Ultrawideband Gated Step Frequency GroundPenetrating Radar. Geoscience and Remote Sensing, IEEE TRANSACTIONS ON (Volume:50, IsSue: 1 )

\section{Authors' Profiles}

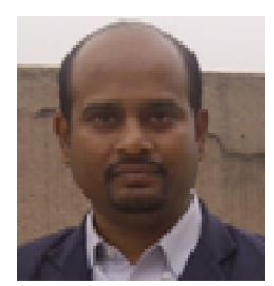

Mr. Subhankar Shome: Born in 1983 at Dumdum, West Bengal, INDIA. He received his B.Sc. (Electronics) from Calcutta University in the year 2005 and received M.Sc. degree from Sikkim Manipal University in the year of 2012 also Pursuing his M.Tech degree from the same University in Digital Electronics \& Advance Communication. $\mathrm{He}$ has been associated with the Sikkim Manipal Institute of Technology, India, from last 8 years, where he is currently working as 
Junior Engineer in the department of Electronics \& Communication Engineering. His area of interest is Broadb and Wireless Communication, Remote Sensing, and Microprocessor \& Microcontroller.

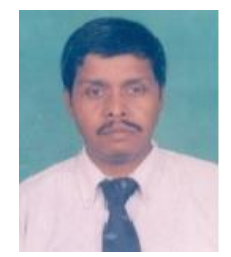

Dr. Rabindranath Bera is a Professor and Ex- Dean (R\&D) in Sikkim Manipal University and Ex- Reader of Calcutta University, West Bengal, India. B.Tech, M.Tech and Ph.D from Radio-Physic \& Electronics, Calcutta University. His Research areas are Digital Radar, RCS Imaging, 4G Communication, Radiometric remote sensing. $\mathrm{He}$ has published a large number of papers in different national \& international Conferences and Journals.

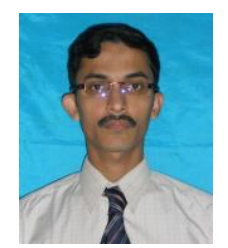

Mr. Samarendra Nath Sur: Born in 1984 at Hooghly, West Bengal, INDIA $\mathrm{He}$ received his M.Sc. (Electronics Science) from Jadavpur University in the year 2007 and M.Tech from Sikkim Manipal University in 2012. He is currently working towards the Ph.D degree in Electronics and Communication Engineering at NIT, Durgapur. Since 2008, he has been associated with the Sikkim Manipal Institute of Technology, India, where he is currently an Asst.Professor in the department of Electronics \& Communication En gineering. Broadband Wireless Communication Advanced Digital Signal Processing and Remote Sensing are the area of specializations.

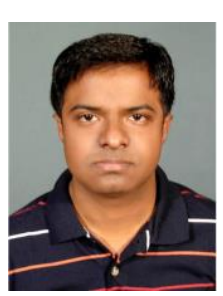

Mr. Rabi Adhikary, BSc. (Electronics), BTech in Electronics \& Communication Enginnering, Serving as an Assistant. Engineer in Electronics \& Communication Dept, Surendra Institute of Engineering \& Management, West Bengal, India. He has presented many papers in various conferences and published referred journals.He has more than 5 years of experience in technical work. His area of interest in Signal Processing and Soft Computing.

How to cite this paper: Subhankar Shome, Rabindra Nath Bera, Samarendra Nath Sur, Rabi Adhikary,"Moving Target Detection and Doppler Extraction Using Digital Spread Spectrum Radar", International Journal of Intelligent Systems and Applications(IJISA), vol.6, no.10, pp.47-54, 2014. DOI: 10.5815/ijisa.2014.10.07 